Research Article

\title{
An Autonomous Waist-Mounted Pedestrian Dead Reckoning System by Coupling Low-Cost MEMS Inertial Sensors and GPS Receiver for 3D Urban Navigation
}

\author{
Jin-feng Li*, Qing-hui Wang, Xiao-mei Liu and Mu-yuan Zhang \\ College of information engineering, Shenyang university of chemical technology, Shenyang,110142, China
}

Received 23 February 2013; Accepted 5 March 2014

\begin{abstract}
Global positioning system (GPS) offers a perfect solution to the 3-dimension(3D) navigation. However, the GPS-only solution can't provide continuous and accurate position information in the unfavourable environments, such as urban canyons, indoor buildings, dense foliages due to signal blockage, interference, or jamming etc. A pedestrian dead reckoning (PDR) system integrating the self-contained inertial sensors with GPS receiver is proposed to provide a seamless outdoor/indoor 3D pedestrian navigation. The MEM sensor module attached to the user's waist is composed of a 3-axis accelerometer, a 3-axis gyroscope, a 3-axis digital compass and a barometric pressure sensor, which doesn't rely on any infrastructure. The positioning algorithm implements a loosely coupled GPS/PDR integration. The sensor data are fused via a complementary filter to reduce the integral drift and magnetic disturbance for accurate heading. The four key components of the PDR algorithm: step detection, stride length estimation, heading and position determination are described in detail and implemented by the microcontroller. The step is detected using the accelerometer signals by the combination of three approaches: sliding window, peak detection and zero-crossing. The step length is estimated using a simple linear relationship with the step frequency. By coupling the step length, azimuth and height, 3D navigation is achieved. The performance of the proposed system is carefully verified through several field outdoor and indoor walking tests. The positioning errors are below $3 \%$ of the total traveled distance. The main error source comes from the orientation estimation. The results indicate that the proposed system is effective in accurate tracking.
\end{abstract}

Keywords: pedestrian dead reckoning (PDR), indoor positioning, inertial navigation system, complementary filter, sensor fusion

\section{Introduction}

Pedestrian tracking and navigation is extensively applied in military, scientific, and commercial applications and has attracted significant attentions in the recent years. Global positioning system (GPS) has widely been used in the most open sky environment, which can provide accurate velocity, time and 3-dimensional absolute position information from a few meters to centimeters ${ }^{[1][2]}$. However, due to the effects of the signal attenuation, satellite geometric distribution and multipath ${ }^{[3]}$, the accuracy of GPS signal is degraded in thick forest, dense urban canyons, and indoor buildings ${ }^{[4]}$. It is difficult to obtain a seamless indoor/outdoor positioning for any GPS-only technology ${ }^{[5]}$.

To bridge the outages of GPS signal, the effective solution is to integrate the GPS information with other position approaches. There are two main methods for indoor positioning. One method is to utilize a local network mounted at the tested locations (beacon-based method), such as ultrasound, infra-red, WiFi, UWB, RFID, Zigbee, vision and so on ${ }^{[6][7][8]}$. However, the deployment of the local network based on infrastructures is generally difficult and time-consuming ${ }^{[1]}$. The second method is to utilize the deadreckoning methods with the self-contained sensors mounted on the person to locate, which is termed as pedestrian deadreckoning (PDR). The PDR is a preferable solution in pedestrian navigation since it does not require any outside preinstalled infrastructures. The combination of inertial sensors and GPS receiver will achieve high accurate pedestrian positioning in both outdoor and indoor environments ${ }^{[9][10]}$.

From a known starting point, the PDR solution integrates the user's step length and orientation estimation at each detected step to compute the user's relative position. The low-cost, small size and weight inertial measurement units (IMU) are required in consideration of it being easily attached to the user's body. The Micro-Electro- Mechanical (MEM) inertial sensors are suited. The MEM inertial sensors, such as accelerometers, gyroscopes, magnetic sensors and so on, are integrated into the PDR system to provide the information for estimating the position. Nevertheless, cheap MEM sensors are sensitive to drift errors. And small errors of the gyroscope signals can accumulate into large angular errors in the orientation. Thus, the suitable hardware and the corresponding algorithm of the PDR system should be carefully designed to improve the PDR positioning accuracy ${ }^{[3][6][7]}$.

The PDR system can be mounted on the user's waist, leg, foot ${ }^{[11]}$ or even the head. The waist location is near the gravity center of the human and is the most reliable position for heading estimation ${ }^{[6]}$. The waist-mounted PDR approach can identify the motions by using only a IMU ${ }^{[12]}$. 
This paper proposes a pedestrian dead reckoning system, which integrates a GPS receiver, a 3-axis accelerometer, a 3axis gyroscope, a 3-axis digital compass and barometric pressure sensor. The wearable IMU is mounted on the user's waist. The positioning algorithm implements a loosely coupled GPS/PDR integration. The inertial sensor data are fused by a complementary filter. The paper is organized as following. Section 2 introduces the PDR system. The data collection methods, step detection, stride length estimation, heading and position determination are described in section 3. The filtering algorithm will also be discussed in detail. Section 4 performs an evaluation of several outdoor and indoor localization tests. The experimental results are presented to demonstrate the effectiveness of the system. Conclusions are given in section 5 .

\section{System Description}

To select proper sensors, many factors, such as size, weight, cost, dynamic range, sampling rate, bias and power consumption, should be considered ${ }^{[1]}$. The inertial sensor module integrates a 3-axis accelerometer and gyroscope MPU6050, a 3-axis digital compass HMC5883 and barometric pressure sensor MS5611. The gyroscope, accelerometer, and digital compass are orthogonally mounted in the sensor module ${ }^{[1]}$. The module is solidly attached to the user's waist. The accelerometer and gyroscope features 16-bit analog-to-digital converters (ADCs) for digitizing the accelerometer and gyroscope outputs, providing $\mathrm{I}^{2} \mathrm{C}$ or SPI port.

The microprocessor chip STM32F407 is the core part of the hardware system. The accelerometer, gyroscope and digital compass communicate with the core via an $\mathrm{I}^{2} \mathrm{C}$ port, while the barometer communicates via an SPI port. The ublox LEA-5 GPS module communicates with the core via a UART interface. The structure of the system is shown in Fig.1. The GPS signal and PDR algorithm are coupled to implement the positioning. When GPS signal is available, the final position and heading of the pedestrian derive directly from the GPS output signal. The GPS information can also be utilized to calibrate the errors of the inertial sensors. When GPS signal is not available, PDR algorithm is triggered to provide the relative position with respect to the last GPS fixed point. Thus, a seamless indoor/outdoor highprecision position is formed. The fusion of digital compass, gyroscope and accelerometer is used to estimate the heading through a complementary filter. The step detection and stride length estimation are performed by accelerometer signal. The position is propagated with the step length and azimuth. The height information is obtained from the barometric pressure sensor. The $3 \mathrm{D}$ navigation is obtained.

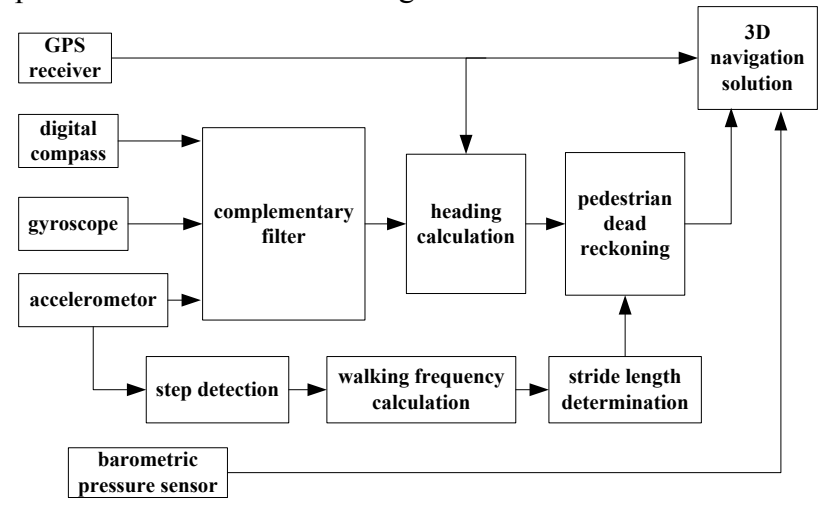

Fig. 1 Architecture of The Proposed Pedestrian Dead Reckoning System

\section{Algorithm}

PDR algorithm contains three main aspects: step detection, step length estimation, heading and position determination ${ }^{[2]}$. The position of the horizontal plane can be deduced from the step length and heading of each step with a known start point.

The low-cost MEM sensors are susceptible to drift errors. The integral errors have serious effects on the angle and will result in large errors which cause obvious deviation in the positioning estimation. It is an essential task for PDR algorithms to reduce the bias and correct the position tracking during walking ${ }^{[1]}$.

In this section, the output signals of the sensors are analyzed firstly. Then, the approaches of step detection, step length estimation and heading determination are discussed. The fusing algorithm using the complementary filter for acquiring the high-precision heading information is described. Finally, 3D position estimation is obtained.

\subsection{Captured Signals}

The sensor module is mounted on the user's waist. The circuit is configured to capture the sensor signals at a frequency of $100 \mathrm{~Hz}$. Fig. 2 shows the raw sensor signals acquired in a normal walking test. The route is 60 -meter long with three 90 degree turns, which requires 81 steps. The step occurrences can be clearly seen from the signal of accelerometer. The 90 degree turns visualize in the signals of gyroscope and digital compass obviously. From Fig. 2, it is known that the step occurrences and step length estimation can be gained from the accelerometer measurement, and the user's azimuth can be determined from the digital compass and gyroscope.
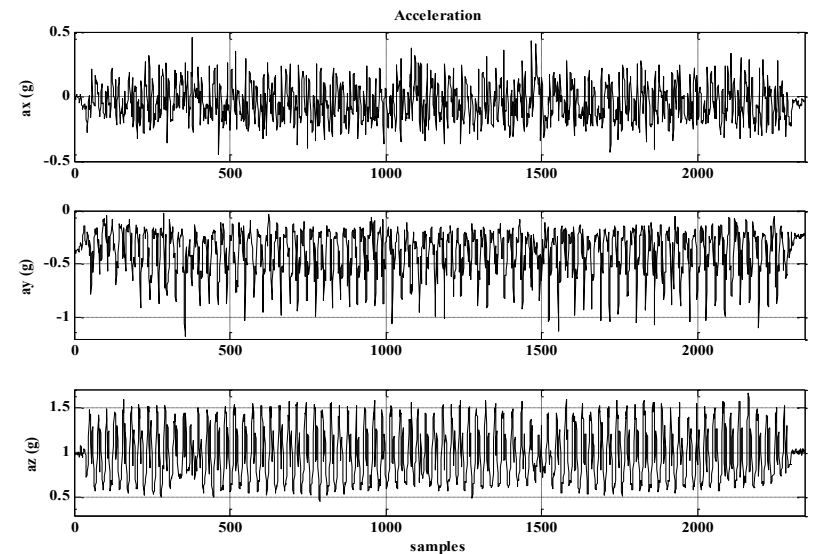

(a)
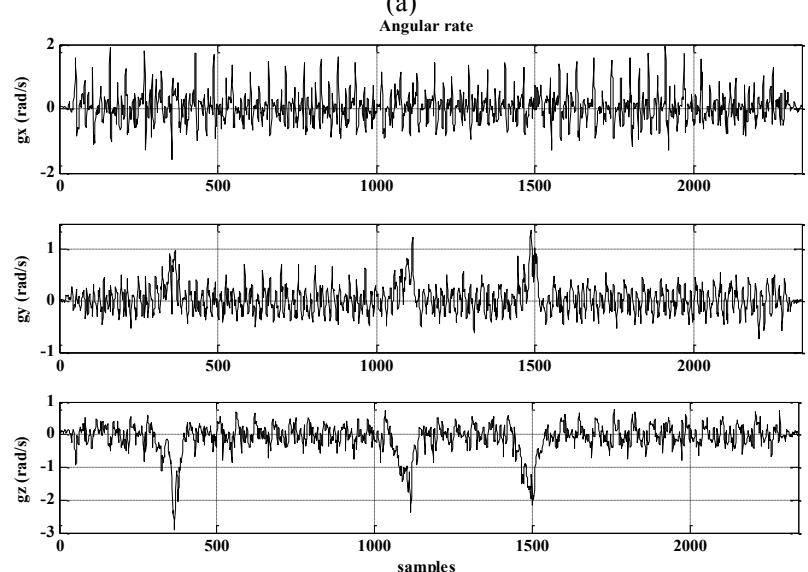

(b) 

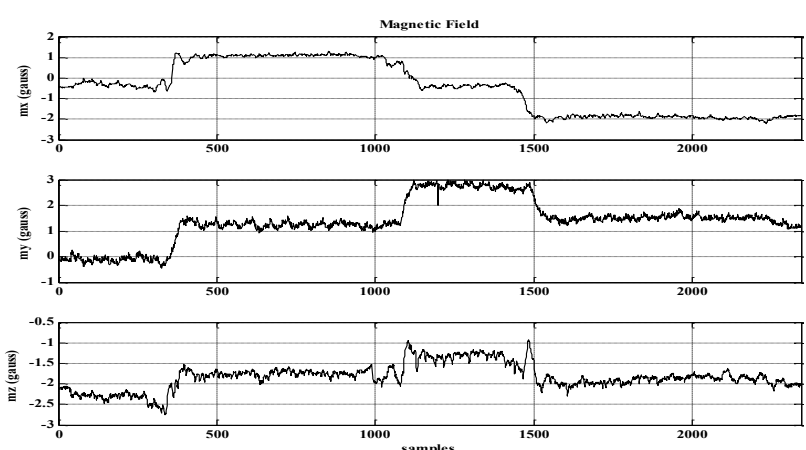

(c)

Fig. 2 Raw Sensor Data from a Test with three 90 Degree Turns (a) Accelerometer (b) Gyroscope and (c) digital compass readings

\subsection{Step Detection}

The step detection is a key element of the PDR algorithm. When a pedestrian is walking, the velocity of a foot periodically changes from stationarity, acceleration, deceleration to stationarity again during every step ${ }^{[1]}$. The accelerometer signal is different due to the various placements of accelerometer on the user's body.

Many kinds of approaches are developed for detecting the step occurrences, such as zero-crossing, peak detection, auto-relation, stance phase detection, zero-velocity update and so on. Which methods adopted depend on the mounted place of the sensor on the user's body ${ }^{[2]}$.In this paper, the accelerometer is mounted on the user's waist. The proposed method combines zero-crossing, peak detection and time restriction for step detection. The steps are identified by comparing the peak with the threshold and taking the minimum step period into account ${ }^{[3]}$.

The 3-axis accelerometer measures the person's forward, lateral and vertical acceleration respectively. All the three axes of the acceleration signal display the cyclical character during walking. The $z$-axis acceleration data shows the character of gait most significantly. The total acceleration data derived from three axes is used to identify the step occurrences in this paper. The magnitude for every sample is:

$a=\sqrt{a_{x}^{2}+a_{y}^{2}+a_{z}^{2}}$

The raw signals of the accelerometer is a little disordered due to the oscillation of the user's body, residual sensor bias and some noise introduced while walking. The disorder may influence the determination of one step's peak, the step length and even cause under or over counting the steps. In order to improve the performance, a smoothing filter is adopted to reduce the effect of disturbance ${ }^{[3]}$.

$a_{k}=\sum_{i=k-L}^{k+L} a_{i}$

where

$2 \mathrm{~L}+1$ is the length of the smoothing filter

From the start point of a stride, the three adjacent values are compared to find a maximum in the local area. In order to eliminate the effects of disturbance, a threshold is employed to validate the peak. If the maximum exceeds the predefined threshold, a valid new peak is identified. The time interval of the new peak value with the peak of the most recent step is computed. If the time interval is greater than the time threshold ${ }^{[13]}$, a new step is detected.The end point of the step is found by zero-crossing ${ }^{[2]}$.

\subsection{Stride Length Estimation}

The step length estimation is a necessary component of the PDR algorithm to calculate the position of the pedestrian. Three main methods may be used ${ }^{[2]}$.One method is defining the step length as a constant or several constants varying with ground condition and walk frequency. The method is inaccurate due to the fact that the step length of a person is a time-varying process. The constant step length will accumulate into big error. The second method is utilizing the artificial intelligent model, such as artificial neural network model, fuzzy logic model and so on. The method can estimate the step length accurately. However, the method is so complex that it is difficult to realize by the microcontroller. Another method is developing a linear model between the step length and the step frequency. This is an effective method. When the step frequency is between 1.35 and 2.45 , the linear relationship can be expressed as ${ }^{[3]}$ :

$S=0.4504 f-0.1656$

where

$f$ is the frequency

$S$ is the stride length

The time interval between the two valid peaks of the steps is the step period, by which the step length can be assessed.

\subsection{Heading Estimation}

The heading estimation is the very important element of PDR algorithm. The heading can be directly captured from the digital compass with respect to magnetic north. However, the digital compass is prone to suffer from unpredictable perturbation of magnetic field ${ }^{[2]}$. The dynamic response characteristic is poor. On the other hand, the gyroscope has good dynamic response characteristic. However, the drift of the gyroscope generates big accumulative error during computing. Consequently, the performance of gyroscope and digital compass can be complemented in the frequency domain. The complementary filter can be designed to fuse the sensor data to increase the accuracy and dynamic performance. The proposed algorithm structure of the complementary filter is shown in Fig. 3.

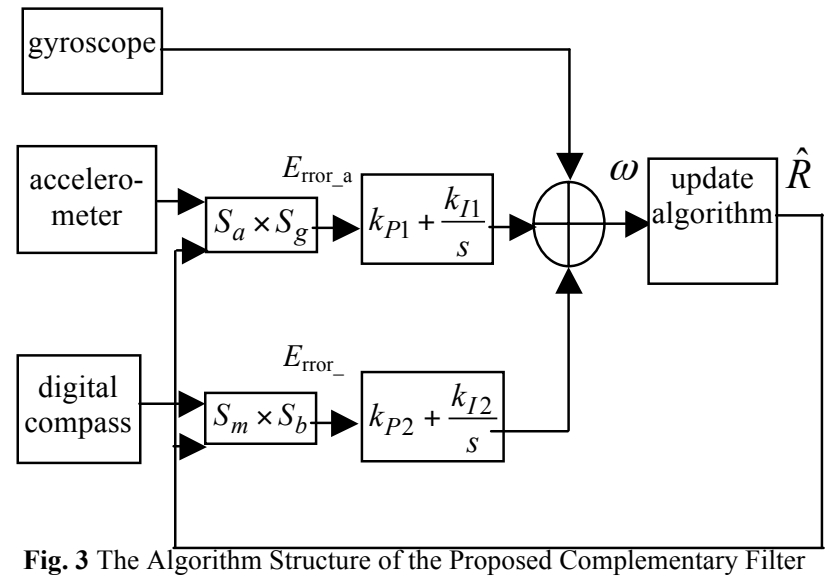


The Euler angle representation is used to develop the algorithm to track the heading. The initial Euler angle can be obtained from the accelerometer and digital compass data. The roll, pitch and yaw of the body can be expressed as:

$$
\begin{aligned}
& \phi=\arctan \frac{a_{y}}{a_{z}} \\
& \theta=\arcsin \frac{a_{x}}{-g} \\
& \psi=\arctan \frac{m_{z} \sin \phi-m_{y} \cos \phi}{m_{x} \cos \theta+m_{y} \sin \theta \sin \phi+m_{z} \sin \theta \cos \phi}
\end{aligned}
$$

where

$\phi, \theta, \psi$ are the roll, pitch and yaw angle respectively

$a_{x}, a_{y}, a_{z}$ are the tested acceleration data of the $x, y, z$ axis

$m_{x}, m_{y}, m_{z}$ are the magnetic field data of the $x, y, z$ axis

$\mathrm{g}$ is the gravity acceleration, $g=\sqrt{a_{x}^{2}+a_{y}^{2}+a_{z}^{2}}$

The initial quaternion can be obtained from the roll, pitch and yaw:

$$
\left[\begin{array}{l}
q_{0} \\
q_{1} \\
q_{2} \\
q_{3}
\end{array}\right]=\left[\begin{array}{c}
\cos \frac{\psi}{2} \cos \frac{\theta}{2} \cos \frac{\phi}{2}+\sin \frac{\psi}{2} \sin \frac{\theta}{2} \sin \frac{\phi}{2} \\
\cos \frac{\psi}{2} \cos \frac{\theta}{2} \sin \frac{\phi}{2}-\sin \frac{\psi}{2} \sin \frac{\theta}{2} \cos \frac{\phi}{2} \\
\cos \frac{\psi}{2} \sin \frac{\theta}{2} \cos \frac{\phi}{2}+\sin \frac{\psi}{2} \cos \frac{\theta}{2} \sin \frac{\phi}{2} \\
\sin \frac{\psi}{2} \cos \frac{\theta}{2} \cos \frac{\phi}{2}-\cos \frac{\psi}{2} \sin \frac{\theta}{2} \sin \frac{\phi}{2}
\end{array}\right]
$$

The predefined reference direction of the field in the earth frame is:

$E_{g}=\left[\begin{array}{llll}0 & 0 & 0 & 1\end{array}\right]$

The reference direction of the field in the sensor frame is:

$$
\begin{aligned}
& S_{g}={ }_{E}^{S} q^{*} \otimes E_{g} \otimes_{E}^{S} q
\end{aligned}
$$

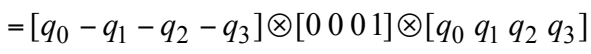

The predefined reference direction of the earth's magnetic field is

$$
\begin{aligned}
& E_{b}=\left[0 \sqrt{h_{x}^{2}+h_{y}^{2}} 0 h_{z}\right] \\
& E_{h}=\left[0 h_{x} h_{y} h_{z}\right]={ }_{E}^{S} q \otimes S_{m} \otimes{ }_{E}^{S} q^{*} \\
& =\left[\begin{array}{llll}
q_{0} & q_{1} & q_{2} & q_{3}
\end{array}\right] \otimes S_{m} \otimes\left[q_{0}-q_{1}-q_{2}-q_{3}\right]
\end{aligned}
$$

The reference direction of the magnetic field in the sensor frame is:

$$
\begin{aligned}
& S_{b}={ }_{E}^{S} q^{*} \otimes E_{b} \otimes{ }_{E}^{S} q
\end{aligned}
$$

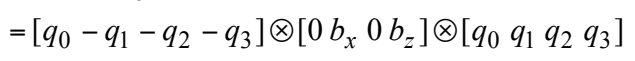

The error between the measured value and the reference value is:

$$
\begin{aligned}
& E_{\text {rror_a }}=S_{a} \times S_{g} \\
& E_{\text {rror_m }}=S_{m} \times S_{b}
\end{aligned}
$$

$\S$ The quaternion is updated at a frequency of $100 \mathrm{~Hz}$ :

$$
\begin{aligned}
& q_{0}=q_{0}+\left(-q_{1} w_{x}-q_{2} w_{y}-q_{3} w_{z}\right) \frac{\Delta t}{2} \\
& q_{1}=q_{1}+\left(q_{0} w_{x}+q_{2} w_{z}-q_{3} w_{y}\right) \frac{\Delta t}{2} \\
& q_{2}=q_{2}+\left(q_{0} w_{y}-q_{1} w_{z}+q_{3} w_{x}\right) \frac{\Delta t}{2} \\
& q_{3}=q_{3}+\left(q_{0} w_{z}+q_{1} w_{y}-q_{2} w_{x}\right) \frac{\Delta t}{2}
\end{aligned}
$$

Finally, the quaternion is transformed to Euler angle for heading used to estimate the position.

\subsection{D Position Estimation}

The position of the pedestrian can be computed by ${ }^{[14]}$.

$$
\begin{aligned}
& E_{k+1}=E_{k}+S_{k} \sin \left(\varphi_{k}\right) \\
& N_{k+1}=N_{k}+S_{k} \cos \left(\varphi_{k}\right) \\
& H_{k+1}=H_{k}+\Delta H_{k}
\end{aligned}
$$

where

the subscript $k$ denotes the value at the step $k$

$E$ denotes the East position coordinate

$N$ denotes the North position coordinate

$H$ denotes the height coordinate

$\varphi$ is the heading with respect to the magnetic north

$\Delta H$ is the height difference

$S$ is the stride distance.

The height information can be calculated from barometer measurements, which is helpful to estimate the floor the user located in.

The relationship between the atmospheric pressure and altitude satisfies:

$$
P=P_{0}\left(1-\frac{H}{44330}\right)^{5.255}
$$

where

$P_{0}$ is the standard atmospheric pressure which value is 1013.25 mbar

$H$ is the height in meter

$P$ is the atmospheric pressure the user located in mbar. 


\section{The Experiments and Results}

To validate the performance of the proposed sensor module and PDR algorithm, several walking tests were conducted at shenyang university of chemical technology (SYUCT). All the inertial sensors and GPS receiver were solidly on the circuit board and attached to the user's waist. The circuit collected the data of inertial sensors and realized the positioning algorithm during multiple walks along several trajectories in both outdoor and indoor environments. The circuit was connected by USB to a notebook computer. The sampling frequency was $100 \mathrm{~Hz}$. The notebook computer was used to record the raw sensor data and displacement measurement results outputting from the circuit. The tests were performed without any support from GPS receiver.

\subsection{Indoor Location Test}

The indoor test was carried out on the $3^{\text {rd }}$ floor corridor in $8^{\text {th }}$ experiment building of SYUCT at normal speed. The tester walked about $174 \mathrm{~m}$ by 260 steps. The indoor test result is shown in Fig. 4. The trajectory contains four 90 degree turns from the start pint $\mathrm{A}$ to the end point $\mathrm{F}$. Three repeated walking are performed to valid the performance of the system. It is obvious that the estimated route between point $\mathrm{A}$ and $\mathrm{B}$ is fit well with the real straight line. Greater heading errors happen at the four 90 degree turns. The greatest length error is approximately $5 \mathrm{~m}$ appearing in the vicinity of the point $\mathrm{D}$, which accounts for a percentage of the total walking distance below $3 \%$.

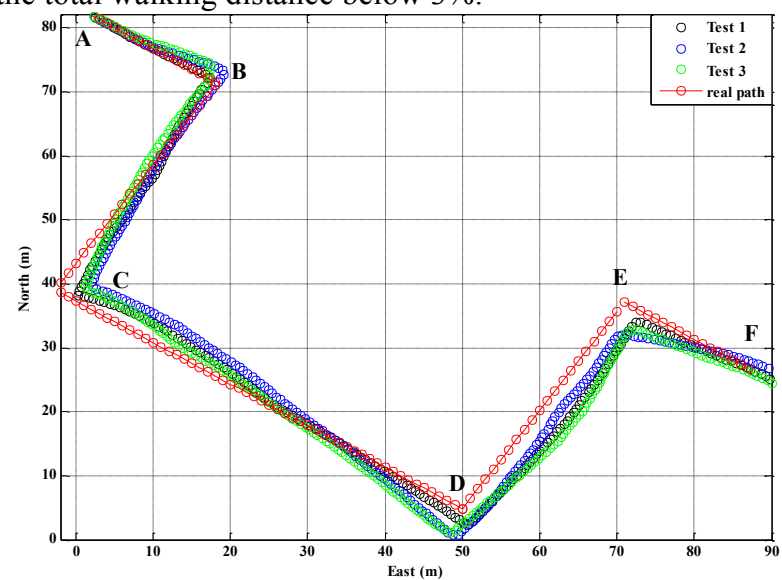

Fig. 4 The Test Result of The Indoor Route

\subsection{Test at different heights}

The estimated trajectory is not restricted to the $X Y$ plane. It can be extended to describe the variation in the vertical direction, such as movements in different floors in a building ${ }^{[15]}$. The height test experiment is performed inside a fivefloor building. The result is shown in Fig. 5. The tester go forward along the $1^{\text {st }}$ floor corridor, then go up to the $2^{\text {nd }}$ floor, and so on, until go up to the $5^{\text {th }}$ floor. Then go down until to the $1^{\text {st }}$ floor. The floor changes can be clearly identified from the curve of Fig. 5. The height difference between the two neighboring floors is about $4 \mathrm{~m}$, which is identical with the real height. The wave fluctuation in every floor is related to the vibration of the test's body.

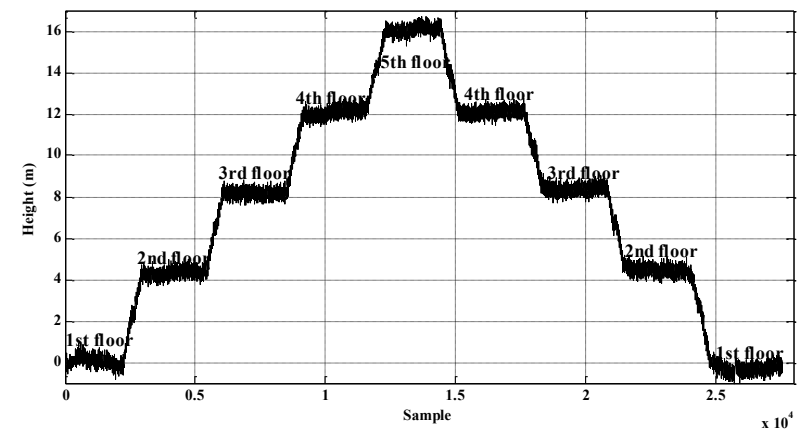

Fig. 5 The Height Test Result in a 5-floor Building

\section{Conclusion}

The hardware integrating GPS receive and low-cost inertial sensors and the corresponding algorithm are proposed for the pedestrian navigation application in indoor environments. No fixed infrastructures are needed in the system. The sensors are restricted to the low-cost, lowweight, low-power MEM sensors. The MEM sensor module is mounted on the user's waist. The step detection, step length estimation, heading and position determination of PDR algorithm are discussed in detail. The heading is determined by fusing the sensor signals using the complementary filter to eliminate the effects of the drift error and magnetic disturbance. The system performance is validated by several field walking tests. The positioning errors are below $3 \%$ of the traveled distance. The results demonstrate the reliability and effectiveness of the system.

\section{References}

1. Chengliang Huang, Zaiyi Liao, Lian Zhao," Synergism of INS and PDR in self-contained pedestrian tracking with a miniature sensor module", IEEE Sensors Journal, 10(8), 2010, pp.1349-1359.

2. Wei Chen, Zhongqian Fu, Ruizhi Chen, Yuwei Chen, Octavian Andrei, et.al," An integrated GPS and multi-sensor pedestrian positioning system for 3D urban navigation", Urban Remote Sensing Joint Event, Shanghai, 2009, pp.1-6.

3. Zuolei Sun, Xuchu Mao, Weifeng Tian, Xiangfen Zhang,“ Activity classification and dead reckoning for pedestrian navigation with wearable sensors", Measurement Science and Technology, 20, 2009, pp.1-10.

4. Widyawan, Martin Klepal, St'ephane Beauregard," A backtracking particle filter for fusing building plans with PDR displacement estimates", Proceedings of The 5th Workshop on Positioning, Navigation and Communication, Hannover, 2008, pp.207-212.

5. Fan Zhang, Xincai Wu, Shang Zhang," GPS location accuracy improvement by WLAN", Computer Modelling and New Technologies", 17(4), 2013.

6. A. R. Jim'enez, F. Seco, C. Prieto, J. Guevara,“ A comparison of pedestrian dead-reckoning algorithms using a low-cost MEMS IMU", 6th IEEE International Symposium on Intelligent Signal Processing, Budapest, 2009, pp.37-42.
7. Antonio Ramón Jiménez Ruiz, Fernando Seco Granja, José Carlos Prieto Honorato, Jorge I. Guevara Rosas," Accurate pedestrian indoor navigation by tightly coupling foot-mounted IMU and RFID measurements", IEEE Transactions on Instrumentation and Measurement, 61(1), 2012, pp.178-189.

8. Hui Liu, Houshang Darabi, Pat Banerjee, Jing Liu," Survey of wireless indoor positioning techniques and systems", IEEE Transactions on Systems, Man, and Cybernetics-Part C: Applications and Reviews, 37(6), 2007, pp.1067-1080.

9. Raúl Feliz, Eduardo Zalama, Jaime Gómez García-Bermejo,“ Pedestrian tracking using inertial sensors", Journal of Physical Agents, 3(1), 2009, pp.35-42.

10. Ge'rard Lachapelle," Pedestrian navigation with high sensitivity GPS receivers and MEMS", Personal and Ubiquitous Computing, 11, 2007, pp.481-488.

11. S Godha, G Lachapelle," Foot mounted inertial system for pedestrian navigation", Measurement Science and Technology, 19, 2008, pp.1-9.

12. Masakatsu Kourogi, Tomoya Ishikawa, Takeshi Kurata," A method of pedestrian dead reckoning using action recognition", Position Location and Navigation Symposium, California, 2010, pp.85-89. 
Jin-feng Li, Qing-hui Wang, Xiao-mei Liu and Mu-yuan Zhang/

Journal of Engineering Science and Technology Review 7 (1) (2014) 9 - 14

13. Yunye Jin, Hong-Song Toh, Wee-Seng Soh, Wai-Choong Wong,“ A robust dead-reckoning pedestrian tracking system with low cost sensors", IEEE International Conference on Pervasive Computing and Communications, Seattle, 2011, pp.222-230.
14. Zexi Liu, Chukwuemeka Aduba, Chang-Hee Won,“ In-plane dead reckoning with knee and waist attached gyroscopes", measurement, 44, 2011, pp.1860-1868.

15. G. Lammel, J. Gutmann, L. Marti, M. Dobler," Indoor navigation with MEMS sensors", Procedia Chemistry 1, 2009, pp.532-535. 